\title{
Effect of Crystalline Microstructure Evolution on Thermoelectric Performance of PEDOT : PSS Films
}

\author{
Xuan Huang, ${ }^{1}$ Liang Deng, ${ }^{1}$ Fusheng Liu, ${ }^{1}$ Qichun Zhang, ${ }^{2,3}$ and Guangming Chen $\mathbb{D}^{1}$ \\ ${ }^{1}$ College of Materials Science and Engineering, Shenzhen University, Shenzhen 518055, China \\ ${ }^{2}$ School of Materials Science and Engineering, Nanyang Technological University, Singapore, Singapore 639798 \\ ${ }^{3}$ Department of Materials Science and Engineering, City University of Hong Kong, Kowloon, Hong Kong SAR, China \\ Correspondence should be addressed to Qichun Zhang; qczhang@ntu.edu.sg and Guangming Chen; chengm@szu.edu.cn
}

Received 9 August 2020; Accepted 30 August 2020; Published 21 January 2021

Copyright (ㄷ 2021 Xuan Huang et al. Exclusive Licensee Beijing Institute of Technology Press. Distributed under a Creative Commons Attribution License (CC BY 4.0).

\begin{abstract}
Although organic polymer thermoelectric (TE) materials have witnessed explosive advances in the recent decade, the molecular mechanism of crystallization engineering of TE performance, even for the most successful polymer of poly(3,4ethylenedioxythiophene) : poly(styrene sulfonate) (PEDOT : PSS), is still far from clear. Here, we deepen the understanding of the role of annealing-induced crystalline microstructure evolution on TE performance of the PEDOT : PSS film with thickness of $10 \mu \mathrm{m}$, which is usually more effective than thin ones in applications. Annealed at optimized temperature of $220^{\circ} \mathrm{C}$, the film displays a power factor of 162.5 times of that of the pristine film before annealing. The enhanced TE performance is associated with the changes of crystallographic and morphologic microstructures, including increased crystallinity and crystal grain size, a domain morphology transformation from granular to crystalline nanofibril, and reduced insulating PSS in the skin layer. These variances facilitate the carrier transport by a transition from 3D to $1 \mathrm{D}$ hopping, reduce the activation energy, and improve the carrier mobility. The mechanism of crystallization engineering reported here can be conceptually extended to other TE polymers and guides the future rational design of preparation principles for organic and composite TE materials.
\end{abstract}

\section{Introduction}

Thermoelectric (TE) materials are irreplaceable in their unique capabilities of harvesting low-grade heat and wide applications in TE generators (TEGs), local cooling, eskin and wearable devices, etc. [1-4]. In the recent decade, organic and organic/inorganic composite TE materials have witnessed drastic advances due to their diverse advantages, including solution processability, lightweight, rich source in earth, super flexibility, and highly adjustable molecular structure or component [5-8]. Because of their low thermal conductivities ( $\kappa$, typically between 0.1 and $0.4 \mathrm{~W} \mathrm{~m}^{-1} \mathrm{~K}^{-1}$ ), their TE performance is often evaluated by the power factor $\left(\mathrm{PF}=S^{2} \sigma\right)$ rather than the figure of merit $\left(Z T=S^{2} \sigma T / \kappa\right)$, where $\sigma, S$, and $T$ stand for the electrical conductivity, the thermopower or Seebeck coefficient, and the absolute temperature, respectively. To date, a variety of judicious strategies of both material preparation and flexible device assembly have been developed, and the corresponding TE performances are boosted [9-12]. Never- theless, in-depth research of the underlying molecular mechanism is rather limited. For example, although the crystalline structures of polymers definitely govern the diverse properties and applications of materials, the relationship between crystalline microstructure evolution and TE performance is still not clear.

Among all of the organic polymer TE materials, poly(3,4-ethylenedioxythiophene) (PEDOT), usually its highly aqueous-dispersible polyelectrolyte complex with poly(styrene sulfonate) (PEDOT : PSS), is probably the most frequently studied and the most successful. In order to greatly enhance the TE performance, many preparation techniques have been proposed, including the construction of polymer nanostructure [13-16], solvent treatment [1720], sulfuric acid crystallization [21], doping and dedoping process [22], and fabrication of composites with inorganic particles [23, 24]. Indeed, the high power factor $\left(1270 \mu \mathrm{W} \mathrm{m}{ }^{-1} \mathrm{~K}^{-2}\right)$ [25] and figure of merit (0.58) [26] have already been achieved for PEDOT and its composites, which are almost comparable to the inorganic TE counterparts. 
Regretfully, the enhanced TE performance is simply explained by macromolecular conformation transition, polymer chain expansion into the ordered alignment, and removal of insulating acid of PSS. Little is known for the dependence of PEDOT : PSS TE performance with its crystalline microstructure evolution. The available few reports concentrate on the effect of crystallization on electrical conductivity (not TE performance) for PEDOT thin films (with thickness $\leq 100 \mathrm{~nm}$ ) made by oxidative chemical vapor deposition (OCVD) [27-29] or spin casting [18-20]. Furthermore, note that relatively thick films of micron scale are usually more effective than thin ones in actual applications, for instance, organic solar cells [30] and harvesting the temperature gradient [31-34]. Therefore, a clear understanding toward the role of crystalline microstructure evolution (including the crystal grain size, the crystallinity, the crystal parameter, and the orientation) on TE performance of PEDOT : PSS films, especially the micron-thick, is strongly desired.

Here, we shed light on the annealing-induced crystallization engineering of TE performance for PEDOT : PSS films with thickness of $10 \mu \mathrm{m}$, obtained by filtering the commercialized product of Clevios PH1000 dispersion. First, the TE performance strongly depends upon the film annealing treatments, and the highest TE performance occurs at the optimized annealing temperature of $220^{\circ} \mathrm{C}$. Then, the carrier transport mechanism is discussed based on the variable range hopping (VRH) model and mobility. Subsequently, the crystalline morphology is directly observed by atomic force microscopy (AFM), while the changes of the crystallinity and the crystallite orientation are monitored by two-dimensional grazing-incidence wide-angle X-ray scattering (2D GIWAXS). In addition, the surface-rich crystallization phenomenon and decreased PSS fraction in the skin region are discovered by X-ray photoelectron spectroscopy (XPS). Finally, the underlying mechanism of the role of crystalline microstructure evolution on TE performance of PEDOT : PSS films is discussed.

\section{Experimental Section}

2.1. Materials. The PEDOT : PSS aqueous solution (Clevios PH1000) was purchased from Heraeus Deutschland GmbH. The concentration of PEDOT : PSS was $1.3 \mathrm{wt} \%$, and the mass ratio of PSS to PEDOT was 2.5. Poly(vinylidene fluoride) (PVDF) membranes (pore size: $0.2 \mu \mathrm{m}$; Jinteng Technology Co. Ltd.) were used directly. Deionized water was used in all of the experiments.

2.2. Freestanding Film Preparation and Subsequent Annealing. First, PEDOT : PSS aqueous solution $(2 \mathrm{~mL})$ was directly vacuum filtered onto a porous PVDF membrane and then dried under vacuum at $60^{\circ} \mathrm{C}$ for $12 \mathrm{~h}$, affording flexible and freestanding PEDOT : PSS nanofilms. The thickness of the film is around $10 \mu \mathrm{m}$. Then, the obtained PEDOT : PSS films were thermally annealed for $12 \mathrm{~h}$ at a desired temperature under nitrogen atmosphere in a tube furnace (NBD-O1200-50IT, NuoBaDi Materials Science and Technology Company).
2.3. Characterizations. The morphologies of the films were observed by an atomic force microscope (AFM, Bruker Dimension ICON). Thermogravimetric analysis (TGA) measurements were carried out with a TGA 55 instrument under nitrogen gas flow at a heating rate of $10^{\circ} \mathrm{C} \mathrm{min}^{-1}$. Powder X-ray diffraction (XRD) measurements were carried out with a multifunction X-ray diffractometer (SmartLab) at a scanning speed of $10^{\circ} \mathrm{min}^{-1}$. In the grazing-incidence wideangle X-ray scattering (GIWAXS) measurements, a Xeuss 2.0 GIWAXS instrument (Genix3D Cu) with a Pilatus 3R 300K detector by Xenocs Beijing Scientific Instrument Technology Co., Ltd., was employed. The samples were tested in vacuum, and the exposure time of $300 \mathrm{~s}$ was applied in integration of $q_{x y}$ and $q_{z}$. Photoemission spectrum (XPS) analysis was conducted by multipurpose X-ray photoemission spectroscopy (Escalab Xi+).

2.4. Measurements of Thermoelectric Performances. The room-temperature electrical conductivities and the Seebeck coefficients were measured by a commercial instrument of a Film Thermoelectric Parameter Test System (MRS-3RT, Wuhan Joule Yacht Science \& Technology Co., Ltd.) using a quasi-steady-state mode. During the measurements, the film sample is rectangular in shape, with a dimension of $2 \mathrm{~cm}$ in length, $0.5 \mathrm{~cm}$ in width, and $10 \mu \mathrm{m}$ in thickness. And the variable-temperature conductivities and the Seebeck coefficients for the PEDOT : PSS film samples were measured using a Film Thermoelectric Parameter Test System (MRS-3, Wuhan Joule Yacht Science \& Technology Co., Ltd.) in the temperature range between $100 \mathrm{~K}$ and $300 \mathrm{~K}$. The carrier mobility $(\mu)$ was measured by the HMS-3000 Hall Measurement System.

\section{Results and Discussion}

Figure 1 displays the schematic illustration of the annealing PEDOT : PSS film (see experimental details in Supporting Information (available here)) and two possible $\pi-\pi$ stacking orientations of PEDOT. In brief, thick films of the pristine PEDOT : PSS were obtained by vacuum-filtrating commercial Clevios PH1000 solution on the porous poly(vinylidene fluoride) (PVDF) membrane (Figure S1). The film with thickness of $\sim 10 \mu \mathrm{m}$ exhibits a high flexibility (Figure S2), whose electrical conductivity and Seebeck coefficient are $1-3 \mathrm{~S} \mathrm{~cm}^{-1}$ and $13-15 \mu \mathrm{V} \mathrm{K}^{-1}$, respectively. Then, the films were thermally annealed at a desired temperature. As a consequence, crystalline films of PEDOT : PSS were achieved, which were further employed to investigate the relationship between crystalline microstructure evolution and TE performance. Two typical crystalline microstructures are presented, where the $\pi-\pi$ stacking orientations of PEDOT chains are parallel (edge-on) or perpendicular (face-on) with respect to the film surface. In order to avoid possible hightemperature degradation of PEDOT : PSS, the annealing temperature range is decided by the thermogravimetric analysis (TGA) test. In Figure S3, the first mass loss section before $150^{\circ} \mathrm{C}$ is ascribed to the removal of water molecules by evaporation. The second stage occurs between $250^{\circ} \mathrm{C}$ and $350^{\circ} \mathrm{C}$, possibly arising from the PSS degradation via the 


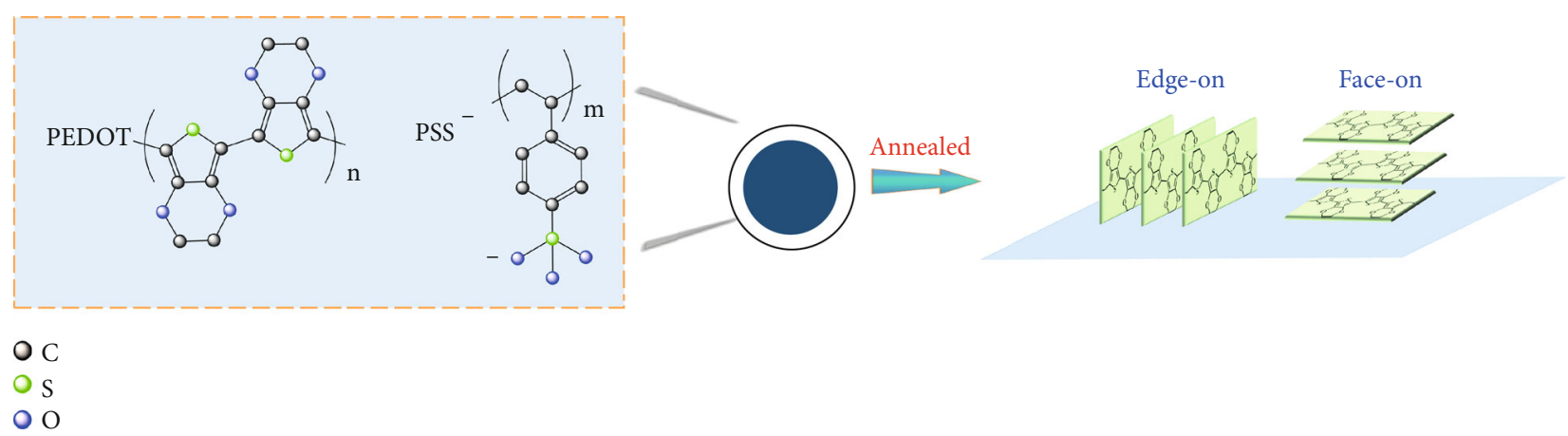

FIgUre 1: Schematic illustration of the annealing of the PEDOT : PSS thick film and two possible $\pi$ - $\pi$ stacking orientations (edge-on and face-on) for crystalline PEDOT macromolecules.

rupture of the sulfonate groups from styrene, and the onset degradation temperature is $275^{\circ} \mathrm{C}[35,36]$. When the temperature is higher than $350^{\circ} \mathrm{C}$, an obvious rupture of the polymer backbone or skeleton breakage takes place, resulting in further mass loss $[37,38]$. Thus, PEDOT : PSS is deduced to reveal an excellent thermal stability below $250^{\circ} \mathrm{C}$, which is consistent with previous studies $[39,40]$. In the present study, the temperature range between 200 and $250^{\circ} \mathrm{C}$ is chosen for annealing treatment.

Figure 2(a) presents the room-temperature TE performances, including the electrical conductivity, the Seebeck coefficient, and the power factor, as a function of annealing temperature for PEDOT : PSS films after being annealed in nitrogen atmosphere for $12 \mathrm{~h}$. Distinctly, the TE performances strongly depend on the annealing temperature. First, all of the annealed films exhibit significantly enhanced electrical conductivities, being approximately two orders of magnitude higher than that of the pristine $\left(1-3 \mathrm{~S} \mathrm{~cm}^{-1}\right)$. Increased annealing temperature leads to a rapid improvement until $220^{\circ} \mathrm{C}$ and a subsequent reduction of the electrical conductivity. At the annealing temperature of $220^{\circ} \mathrm{C}$, the PEDOT : PSS film shows the maximum room-temperature electrical conductivity of $596 \pm 14 \mathrm{~S} \mathrm{~cm}^{-1}$. The other important parameter, the Seebeck coefficient, reveals a similar trend to the electrical conductivity, i.e., first increase gradually and then reduce with annealing temperature. The largest Seebeck coefficient is $23.3 \pm 0.7 \mu \mathrm{V} \mathrm{K}^{-1}$ for the film annealed at $220^{\circ} \mathrm{C}$, being much larger than that of the pristine film $\left(13-15 \mu \mathrm{V} \mathrm{K}^{-1}\right)$ as well. As a consequence, the power factor exhibits the same trend in a curve shape as the electrical conductivity or the Seebeck coefficient. At the optimized annealing temperature of $220^{\circ} \mathrm{C}$, the PEDOT : PSS film displays the highest power factor, reaching $32.5 \pm 2.5 \mu \mathrm{W} \mathrm{m}{ }^{-1} \mathrm{~K}^{-2}$. In sharp contrast, the pristine film has a low power factor of only around $0.2 \mu \mathrm{W} \mathrm{m}^{-1} \mathrm{~K}^{-2}$. In other words, by thermal annealing, the power factor for the PEDOT : PSS thick film is boosted, with a maximum of 162.5 times of that of the pristine film before annealing. Indeed, the power factor of $32.5 \mu \mathrm{W} \mathrm{m}^{-1} \mathrm{~K}^{-2}$ is higher than that of the pristine PEDOT : PSS films reported in many previous investigations [39, 41].

In order to elucidate the underlying molecular mechanism toward the enhancement of TE performance by thermal annealing, the temperature dependence test of the electrical conductivity is conducted in the range of $100-300 \mathrm{~K}$ (Figure 2(b)). For all of the PEDOT : PSS films, their electrical conductivities improve with the increase of annealing temperature, confirming the semiconducting transport nature of PEDOT : PSS at low temperature. Moreover, at a fixed temperature, the electrical conductivities follow the sequence of annealing at $220^{\circ} \mathrm{C}>$ at $240^{\circ} \mathrm{C}>$ the pristine before annealing. To gain more insights into the carrier transport mechanism, the VRH model is employed, which has been extensively applied for polymer TE materials with semiconducting behaviors $[42,43]$. The equation is presented in the following:

$$
\sigma=\sigma_{0} \exp \left[-\left(\frac{T_{0}}{T}\right)^{\alpha}\right],
$$

where $\sigma_{0}$ represents the electrical conductivity at the infinite temperature $(0 \mathrm{~K}), T_{0}$ stands for the characteristic temperature, $T$ is the current temperature, and $\alpha$ equals $1 /(1+D)$ ( $D$ is the dimensionality) [44]. If $\alpha$ is $1 / 4$, the carriers display a three-dimensional (3D) transport mechanism. And if $\alpha$ is $1 / 2$, a $1 \mathrm{D}$ carrier transport can be deduced. As evidenced by Figures 2(c) and 2(d), $\ln \sigma$ shows an essential linear relationship with (c) $T^{-1 / 4}$ for the pristine film and (d) $T^{-1 / 2}$ for the film annealed at 220 or $240^{\circ} \mathrm{C}$. Therefore, we conclude that the carrier transport route varies from $3 \mathrm{D}$ to $1 \mathrm{D}$ direction after annealing, while the annealing temperature has little effect on the carrier transport dimension. Furthermore, the detailed parameters deduced from the VRH model are compared in Table 1 . The room-temperature electrical conductivities for the annealed film samples are $596 \pm 14 \mathrm{~S} \mathrm{~cm}^{-1}$ $\left(220^{\circ} \mathrm{C}\right)$ and $445 \pm 2 \mathrm{~S} \mathrm{~cm}^{-1}\left(240^{\circ} \mathrm{C}\right)$, respectively, much higher than that for the pristine film $\left(1-3 \mathrm{~S} \mathrm{~cm}^{-1}\right)$. Indeed, the pristine film exhibits a metal behavior at temperatures higher than $550 \mathrm{~K}$, as presented in Figure $S 4 . T_{0}$ is an important parameter, characteristic of the activation energies of carrier hopping barriers. Here, the pristine thick film reveals a $T_{0}$ of $119.3 \mathrm{~K}$, which is much lower than that of thin films prepared by spin coating, for instance, $4210 \mathrm{~K}$ for the film with $27 \mathrm{~nm}$ in thickness [42]. After annealing, the $T_{0}$ decreased to $14.3 \mathrm{~K}\left(220^{\circ} \mathrm{C}\right)$ and $17.6 \mathrm{~K}\left(240^{\circ} \mathrm{C}\right)$. Meanwhile, the $\sigma_{0}$ of the pristine $\left(30.0 \mathrm{~S} \mathrm{~cm}^{-1}\right)$ increases to 


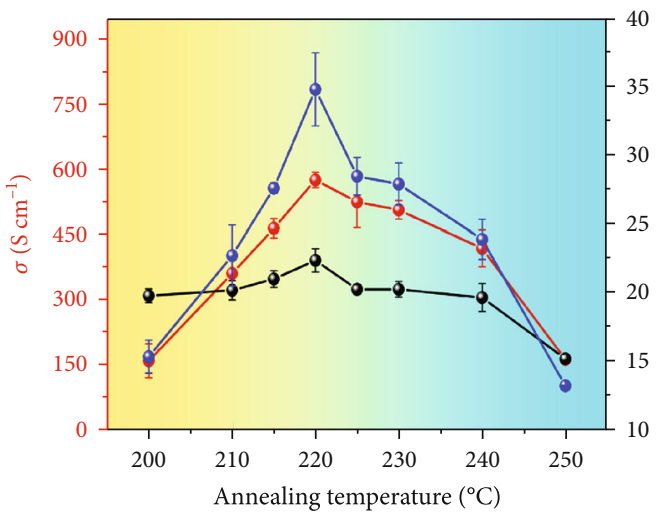

(a)

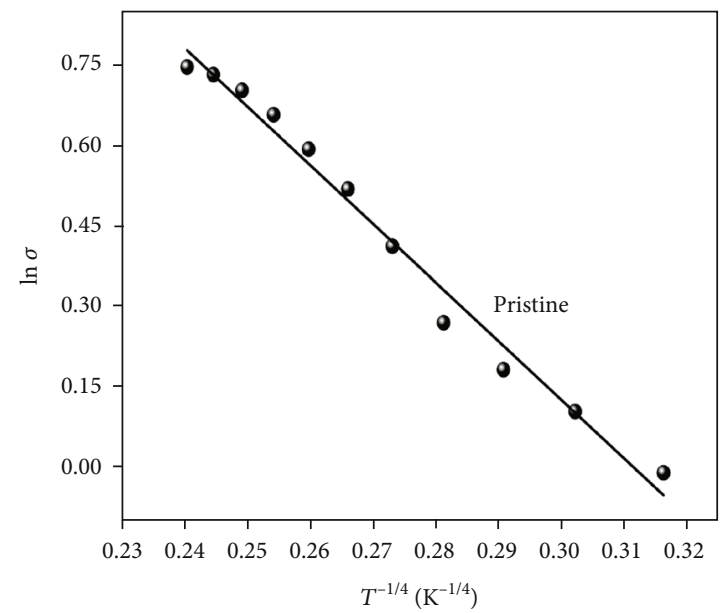

(c)

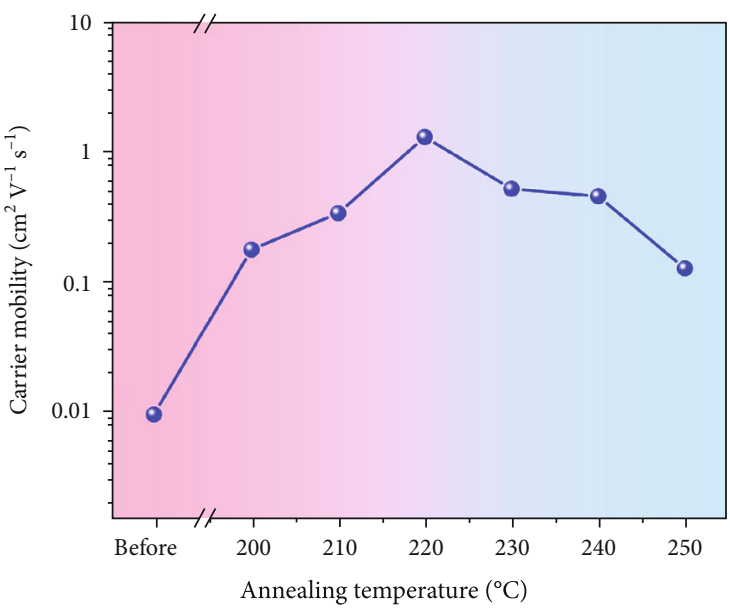

(e)

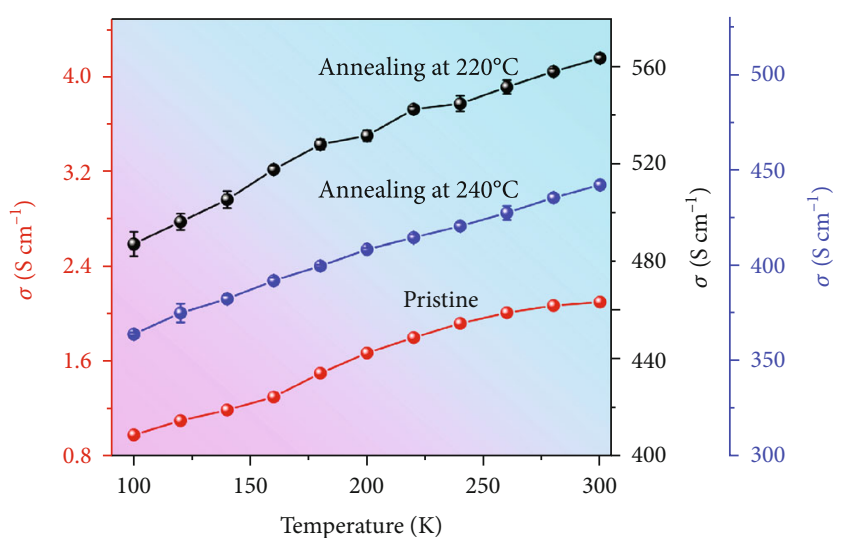

(b)

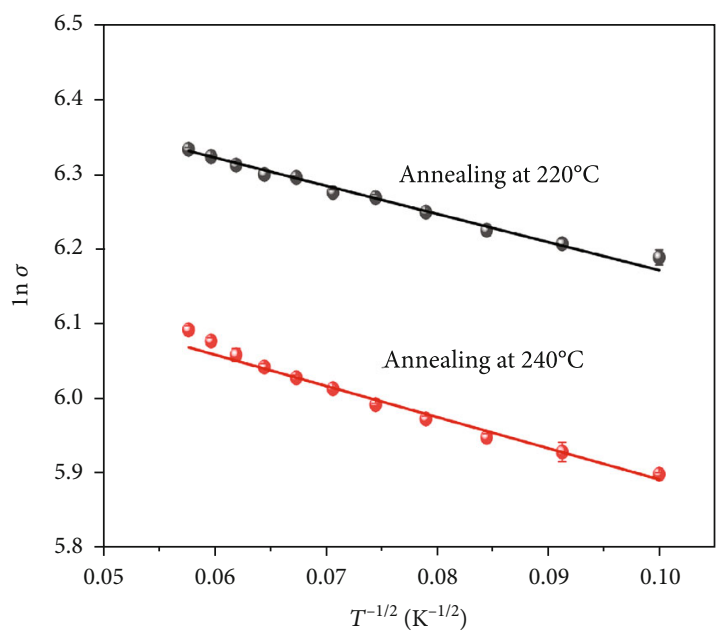

(d)

FIgURE 2: (a) Room-temperature TE performances for PEDOT : PSS films as a function of annealing temperature after being annealed in nitrogen atmosphere for $12 \mathrm{~h}$. (b) Temperature dependences of electrical conductivity for the pristine PEDOT : PSS film before annealing, and the films annealed at 220 or $240^{\circ} \mathrm{C}$. The dependence of $\ln \sigma$ with annealing temperature of (c) $T^{-1 / 4}$ for the pristine PEDOT : PSS film or (d) $T^{-1 / 2}$ for the film after being annealed at $220^{\circ} \mathrm{C}$ or $240^{\circ} \mathrm{C}$. (e) Effect of annealing temperature on room-temperature carrier mobility for the pristine and annealed samples, measured by the Hall effect.

$544.6 \mathrm{~S} \mathrm{~cm}^{-1}$ and $699.2 \mathrm{~S} \mathrm{~cm}^{-1}$ for the film annealed at $240^{\circ} \mathrm{C}$ and $220^{\circ} \mathrm{C}$, respectively. Moreover, the film annealed at $220^{\circ} \mathrm{C}$ displays the lowest $T_{0}$ and the highest $\sigma_{0}$. These demonstrate that thermal annealing can effectively lower the energy barrier and facilitate the carrier variable range hopping, and $220^{\circ} \mathrm{C}$ is the optimized annealing temperature. 
TABLE 1: The detailed parameters of the VRH model for the pristine and annealed PEDOT : PSS films.

\begin{tabular}{lccc}
\hline & Pristine & $\begin{array}{c}\text { Annealing at } \\
220^{\circ} \mathrm{C}\end{array}$ & $\begin{array}{c}\text { Annealing at } \\
240^{\circ} \mathrm{C}\end{array}$ \\
\hline Hopping transport & $3 \mathrm{D}$ & $1 \mathrm{D}$ & $1 \mathrm{D}$ \\
$\sigma$ at $300 \mathrm{~K}\left(\mathrm{~S} \mathrm{~cm}^{-1}\right)$ & $1-3$ & $596 \pm 14$ & $445 \pm 2$ \\
$\alpha(100 \mathrm{~K}-300 \mathrm{~K})$ & 0.25 & 0.5 & 0.5 \\
$T_{0}(\mathrm{~K})$ & 119.3 & 14.3 & 17.6 \\
$\sigma_{0}\left(\mathrm{~S} \mathrm{~cm}^{-1}\right)$ & 30.0 & 699.2 & 544.6 \\
\hline
\end{tabular}

To further elucidate the annealing process, the carrier mobility as a function of annealing temperature is measured by the Hall effect (Figure 2(e)). The carrier mobility of the pristine PEDOT : PSS film is very low, being only $9.37 \times$ $10^{-3} \mathrm{~cm}^{2} \mathrm{~V}^{-1} \mathrm{~s}^{-1}$. After annealing, the carrier mobilities are improved remarkably by at least one order of magnitude. Very interestingly, the curve shape of the carrier mobility in Figure 2(e) is nearly identical to that of the TE performance shown in Figure 2(a). In other words, carrier mobility is critical for TE performance. The whole curve can be essentially divided into two parts. When the annealing temperature is below $220^{\circ} \mathrm{C}$, the carrier mobility increases dramatically with annealing temperature, hence a simultaneous enhancement of the electrical conductivity and the Seebeck coefficient. For the film annealed at $220^{\circ} \mathrm{C}$, the carrier mobility reaches its maximum of $1.27 \mathrm{~cm}^{2} \mathrm{~V}^{-1} \mathrm{~s}^{-1}$, being approximately 136 times of that of the pristine film. And the maxima reach $596 \pm 14 \mathrm{~S} \mathrm{~cm}^{-1}$ and $23.3 \pm 0.7 \mu \mathrm{V} \mathrm{K}^{-1}$, respectively. In other words, the optimized crystallization temperature is $220^{\circ} \mathrm{C}$. A further increase of annealing temperature results in a gradually reduced mobility. Considering reduction of TE performance at annealing temperature over $220^{\circ} \mathrm{C}$, three samples, including the pristine film and the films annealed at 220 or $240^{\circ} \mathrm{C}$, have been chosen for the following study of the role of crystalline microstructure evolution on PEDOT : PSS TE performance.

The morphology feature changes induced by thermal annealing are directly observed by AFM images, as shown in Figure 3. Distinctly, the surface of the pristine film displays small PEDOT spherical nanoparticles (white contrast) connected by PSS (Figure 3(a)). In contrast, Figures 3(b) and $3(\mathrm{c})$ reveal that after the thermal annealing at $220^{\circ} \mathrm{C}$ or $240^{\circ} \mathrm{C}$, a nanofibril-shaped morphology with distinct contrast is evident, and the granular particles of PEDOT : PSS agglomerates disappear. Moreover, the surface morphology is quantitatively estimated by the root mean square (RMS) from the topographic images (Figures $3(\mathrm{~d})-3(\mathrm{f})$ ). The pristine PEDOT : PSS film is relatively uniform and smooth, exhibiting a RMS of $1.19 \mathrm{~nm}$ (Figure 3(d)), which is similar to the previous report $[45,46]$. After annealing at $220^{\circ} \mathrm{C}$ or $240^{\circ} \mathrm{C}$, the RMS increase distinctly to $1.48 \mathrm{~nm}$ (Figure 3(e)) and $1.28 \mathrm{~nm}$ (Figure 3(f)), respectively. This demonstrates that the surfaces become rough for the annealed films due to the increase of the crystallite domain sizes (white contrast). Additionally, the brightness increases, implying the improved crystallinity. Therefore, the AFM images provide a solid proof that the annealing procedure induces a morphology transition from nanospherical granule to wellcrystalline nanofibril, and both crystallinity and crystallite domain dimensions increase. Furthermore, the film annealed at $220^{\circ} \mathrm{C}$ displays the largest crystallite domain size and the roughest surface.

Although the ordered molecular packing of conducting polymers has been widely regarded as the cause to the simultaneous increase of $S$ and $\sigma[47,48]$, the proof of detailed structural information is limited, especially for thick films. Here, large-area mapping of 2D GIWAXS, a powerful technique to monitor the molecular stacking and orientation, has been employed. In Figures 4(a)-4(c), scattering of stacking of alternating PEDOT and PSS $\left(q \sim 0.3 \AA^{-1}\right)$ for all thick films along the $q_{z}$ axis exhibited much higher (100) intensities than that of $q_{x y}$, confirming a dominant preferred orientation with $\pi-\pi$ stacking parallel to the film plane, i.e., edge-on stacking. This is different from previous research of remarkable face-on stacking in thin films [49]. In addition, the scattering intensities follow the sequence of $(a) \ll(c)<(b)$, implying the order of the film crystallinity: the pristine film (a) $\ll$ the film annealed at $240^{\circ} \mathrm{C}(\mathrm{c})<$ the film annealed at $220^{\circ} \mathrm{C}(\mathrm{b})$. This agrees with the above AFM result (Figure 3), demonstrating that annealing enables a drastic improved crystallinity and the film annealed at $220^{\circ} \mathrm{C}$ exhibits the highest crystallinity. In addition to the $2 \mathrm{D}$ spectra, onedimensional (1D) GIWAXS patterns are collected by line scans. In Figure $4(\mathrm{~d})$, the peaks at $0.3 \AA^{-1}$ and $1.8 \AA^{-1}$ are assigned to the stacking of alternating PEDOT : PSS and the $\pi-\pi$ stacking (010), respectively $[21,32]$. Due to the deviance in crystallinity, the film annealed at $220^{\circ} \mathrm{C}$ displays the highest scattering intensities for both peaks, while the patterns for the pristine film are much weaker than either of the annealed ones. In the out-of-plane patterns $\left(q_{z}\right)$ (Figure $4(\mathrm{e})$ ), besides an amorphous halo of PSS, a series of sharp peaks of (100) and (200) reflections are characteristic of lamellar stacking of PEDOT and PSS, while the peak at $q_{z} \approx 1.8 \AA^{-1}$ corresponds to the preferred edge-on $\pi-\pi$ stacking. The deviance in peak intensities agrees with Figure 4(d). Additionally, peak shifts can be recognized, where (100) and (200) reflections for the samples annealed at 220 and $240^{\circ} \mathrm{C}$ increase from 0.23 and $0.52 \AA^{-1}$ to 0.25 and $0.56 \AA^{-1}$, respectively. Hence, the lamellar distances of alternating PEDOT and PSS can be calculated to be 27.3 and $25.1 \AA$ for the samples annealed at 220 and $240^{\circ} \mathrm{C}$, respectively. In case of low crystallinity for the pristine film and the relatively broad peak for the face-on stacking with low content, their stacking distances are not taken into account. Deduced from the (010) reflection, the lamellar $\pi-\pi$ stacking distances (real space distances) are calculated to be 3.55 and $3.50 \AA$ for the samples annealed at 220 and $240^{\circ} \mathrm{C}$ (Table S1), respectively, which is similar to the reported data ( 3.5 $\AA$ ) for the solution-processed PEDOT : PSS film [21]. And the crystal domain size for the film annealed at $220^{\circ} \mathrm{C}(4.7 \mathrm{~nm})$ is a little bit larger than that at $240^{\circ} \mathrm{C}$ $(4.4 \mathrm{~nm})$, contributing to the enhanced thermoelectric performance as well.

The annealing also induces a variance in the PEDOT: PSS composition ratio, as evidenced by XPS analyses shown 


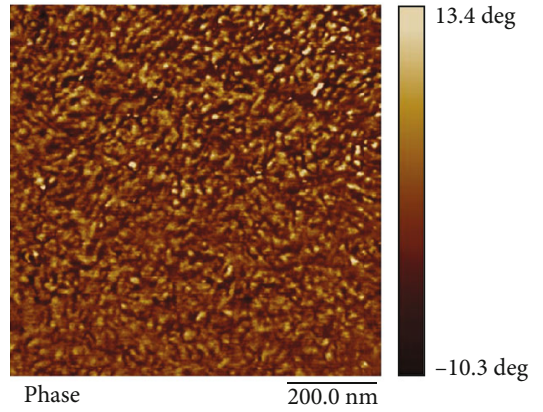

(a)

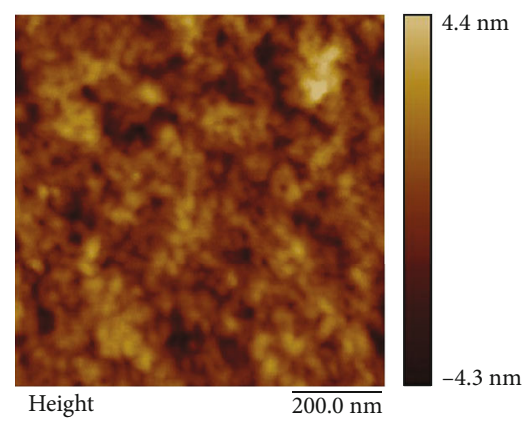

(d)

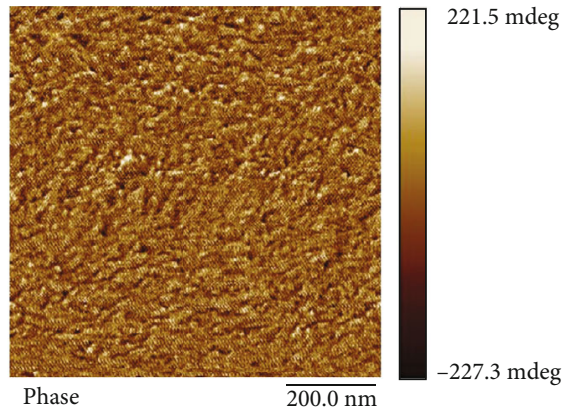

(b)

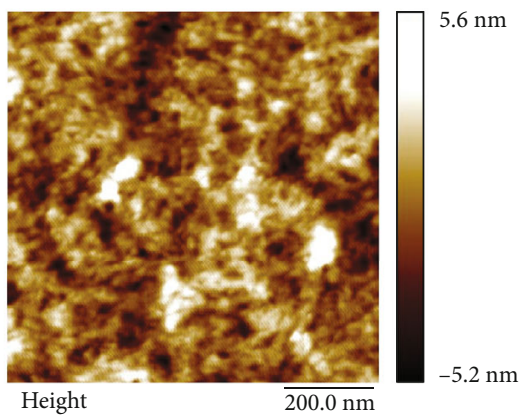

(e)

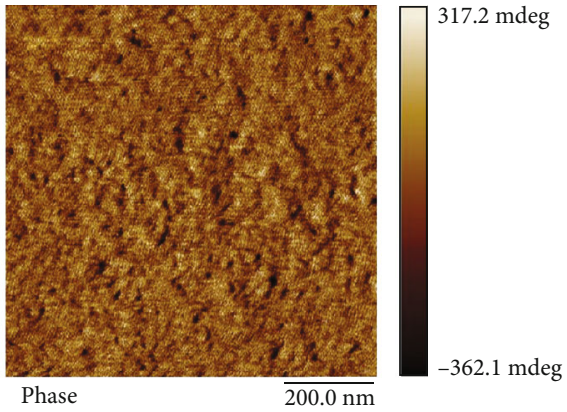

(c)

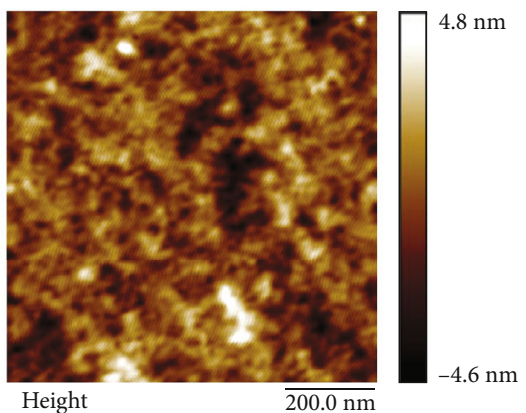

(f)

FIgURE 3: AFM observations of the $(a-c)$ phase images and $(d-f)$ topographic images for $(a, d)$ the pristine PEDOT : PSS film and the annealed film at $(\mathrm{b}, \mathrm{e}) 220^{\circ} \mathrm{C}$ or $(\mathrm{c}, \mathrm{f}) 240^{\circ} \mathrm{C}$.

in Figure 5. The full spectra of the pristine film and the annealed (at 220 and $240^{\circ} \mathrm{C}$ ) PEDOT : PSS films (Figure 5(a)) are identical in a curve shape and clearly demonstrate the existence of the $\mathrm{C}, \mathrm{S}$, and $\mathrm{O}$ elements. The corresponding peak fittings of core level spectra (S 2p) are presented in Figures $5(\mathrm{~b})-5(\mathrm{~d})$. The $S 2 \mathrm{p}$ peak can be divided into two sections: the peaks between 166 and $168 \mathrm{eV}$ can be assigned to the sulfur atoms from the sulfonate groups in PSS, while the peaks in the range of $162-164 \mathrm{eV}$ are relative to those from the thiophene groups in PEDOT $[47,48]$. Both peaks involve the contributions from a spinsplit doublet, i.e., $S 2 p_{3 / 2}$ and $S 2 p_{1 / 2}$. Furthermore, the molar ratio of PEDOT : PSS can be estimated from the peak areas of PEDOT and PSS. After annealing, the PEDOT : PSS ratio reduces obviously from $1: 2.8$ (the pristine) to $1: 2.1$ (annealed at $240^{\circ} \mathrm{C}$ ) and even $1: 1.7$ (annealed at $220^{\circ} \mathrm{C}$ ). Note that all of the characterization techniques including XPS, AFM, and GIWAXS reveal the information on the film surface layer. In other words, the thermal annealing enables the motion of PEDOT molecules from the inner region to the outer film surface. The reason may be assigned to macromolecular crystallization, and preferred orientation usually tends to take place in the skin layer rather than the core region $[47,48,50]$.

For semicrystalline polymers, the crystalline microstructure evolution is vital for carrier transport and hopping, hence affecting the TE performance. The hopping mainly means the "coarse-grained" way from one crystallite site to another, instead of one-to-neighbor atomic sites in the amorphous phase [27]. It is an effective method to reduce the carrier transport barriers between crystallized regions. Taking all evidences together, the annealinginduced PEDOT : PSS crystalline structure evolution is schematically displayed in Figure 6, which shed light on the mechanism understanding of the enhanced TE performance. The pristine PEDOT : PSS thick film reveals a low crystallinity, a small crystallite size, a mixture of relatively more edgeon crystallites and less face-on one, and a high content of PSS in the skin layer. These features contribute to a very low mobility $\left(9.37 \times 10^{-3} \mathrm{~cm}^{2} \mathrm{~V}^{-1} \mathrm{~s}^{-1}\right)$, a relatively high hopping barrier $\left(T_{0}=119.3 \mathrm{~K}\right)$, and a $3 \mathrm{D}$ transport mechanism of carrier hopping. As a consequence, the pristine film exhibits a very low TE performance $\left(\sim 0.2 \mu \mathrm{W} \mathrm{m}^{-1} \mathrm{~K}^{-2}\right)$. The following factors of the annealing-induced crystalline microstructure evolution may contribute greatly to the enhanced TE performance for the annealed PEDOT : PSS thick films:

(1) The thermal annealing induces significant increases of both crystallinity and crystallite grain size, as well as a domain morphology transformation from nanospherical granule to well-crystalline nanofibril. Due to the formation of the ordered crystalline structure, the "coil-like" PEDOT chains are expanded or stretched to straighter or "rod-like." The increased crystallite size and the occurrence of domain nanofibril morphology reduce the distances between adjacent crystallite grains and benefit the overlap between their total wavefunctions [21, 50]. Thus, the carrier hopping among adjacent crystallites are facilitated by the increase of effective carriers' 


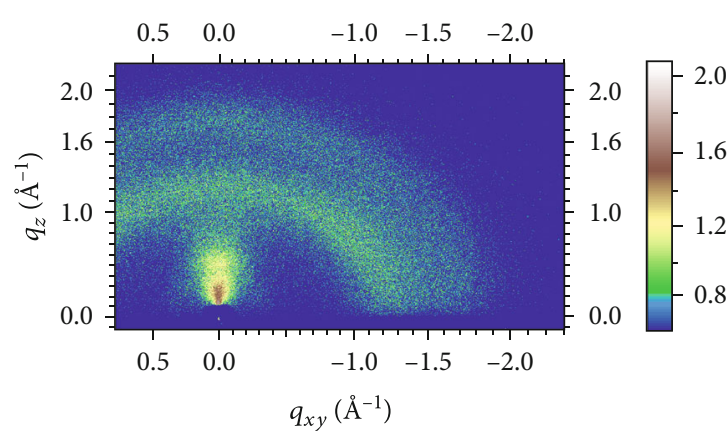

(a)

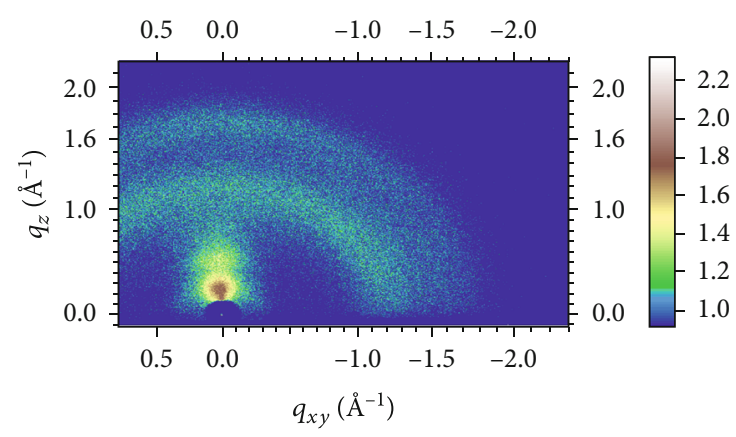

(b)

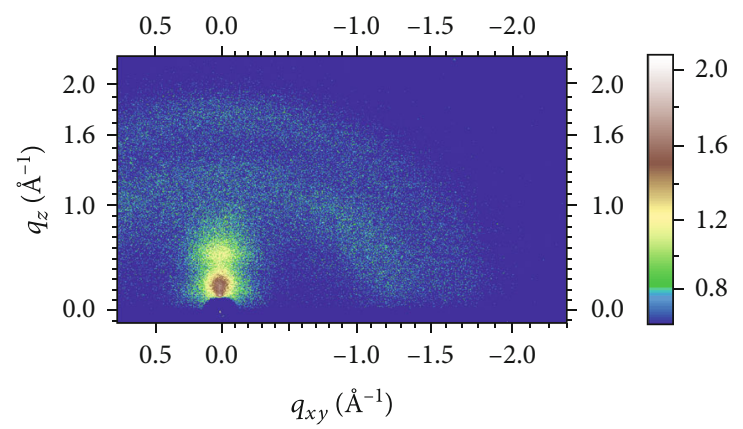

(c)

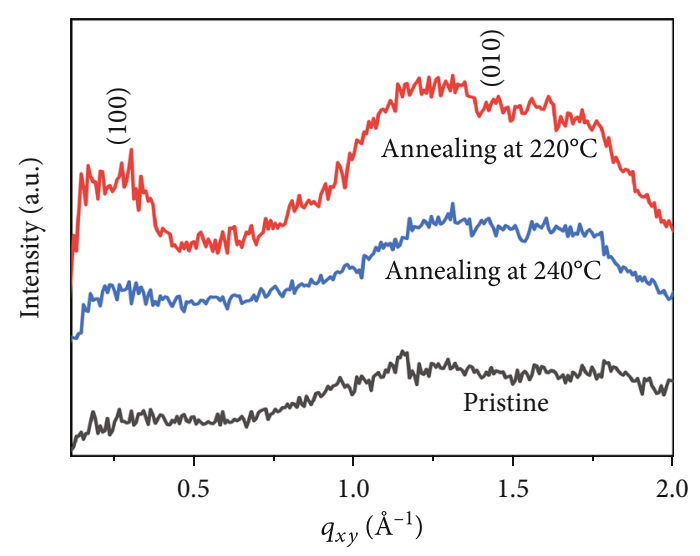

(d)

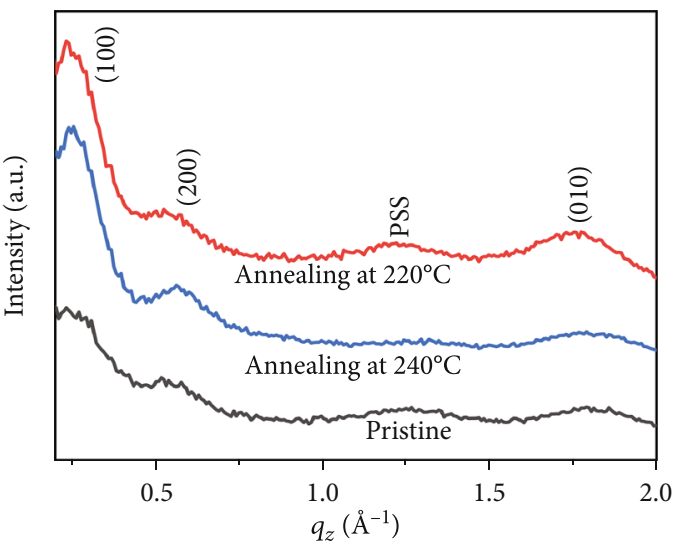

(e)

Figure 4: 2D GIWAXS patterns of (a) the pristine PEDOT : PSS film and the film being annealed at (b) 220 or (c) $240^{\circ} \mathrm{C}$. Linearly offset $1 \mathrm{D}$ GIWAXS patterns of the pristine and annealed PEDOT : PSS films with $(\mathrm{d})$ in-plane $\left(q_{x y}\right)$ or $(\mathrm{e})$ out-of-plane $\left(q_{z}\right)$ scattering geometry.

transport pathways of both the intra- and interadjacent macromolecular chains

(2) Since the interchain coupling has a remarkable impact on the electronic transport in PEDOT : PSS films, the dominant preferred orientation of $\pi-\pi$ edge-on stacking dramatically facilitates the carrier hopping and transport along the film plane direction, which is superior to the face-on stacking in thin films $(\leq 100 \mathrm{~nm})$, thus favoring the carrier hopping and transport of intercoupling macromolecular chains

(3) The high fraction of PEDOT crystal domains and reduced insulating PSS in the film skin layer resulted from skin-layer preferred crystallization, helped the carrier hopping, and improved the TE performance. This phenomenon has not been observed in thin films $(\leq 100 \mathrm{~nm})$ of PEDOT : PSS
These main factors will significantly reduce the hopping activation energies for carrier transportation, resulting in a remarkable increase of carrier mobility and TE performances. In addition, the increase of annealing temperature enhances the macromolecular segment motions of both the formation of the ordered crystalline structure and also the relaxation of disordered-to-ordered transition. In the present study, the optimized annealing temperature is $220^{\circ} \mathrm{C}$, and the corresponding annealed film exhibits the highest mobility and TE performances. A further increase of annealing temperature leads to a reduced crystallinity, a reduced crystallite size, and an increased PSS content in the film skin region, hence reducing the carrier mobility and the TE performances.

\section{Conclusions}

In summary, we obtain the insights into a clear understanding of the mechanism of the role of annealing- 


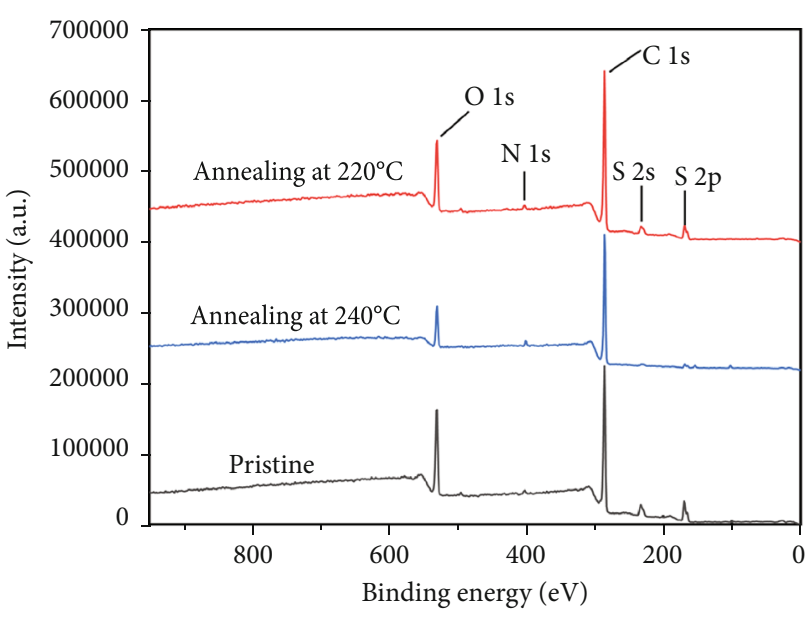

(a)

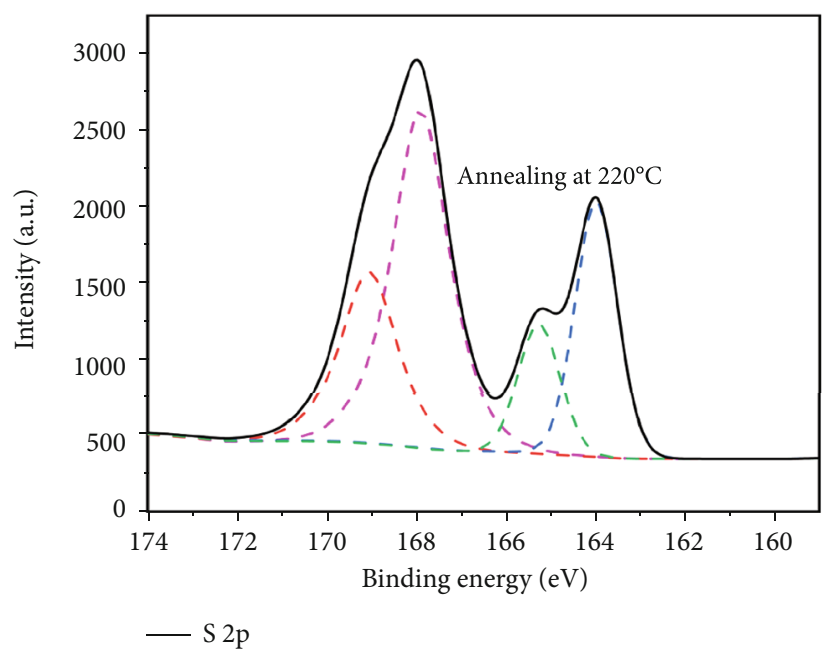

(c)

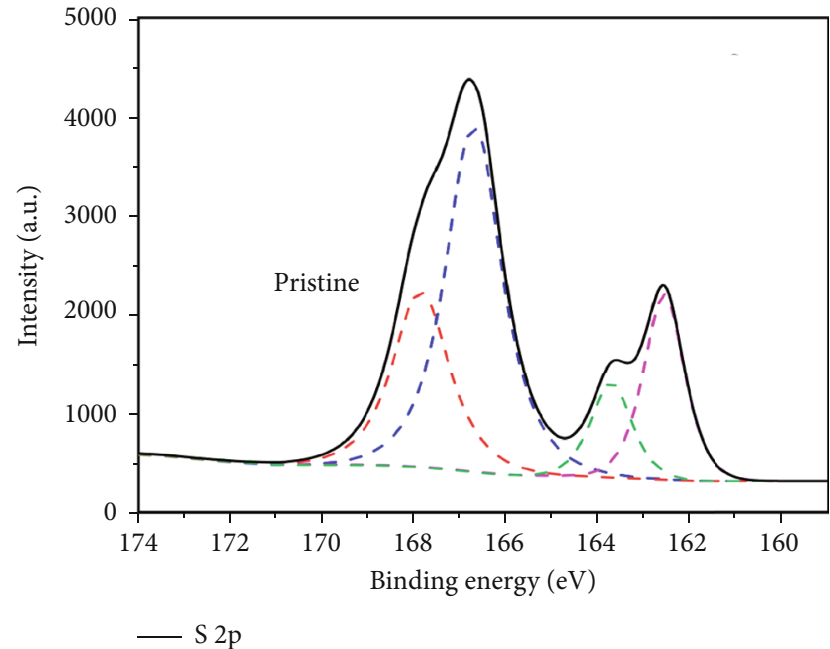

(b)

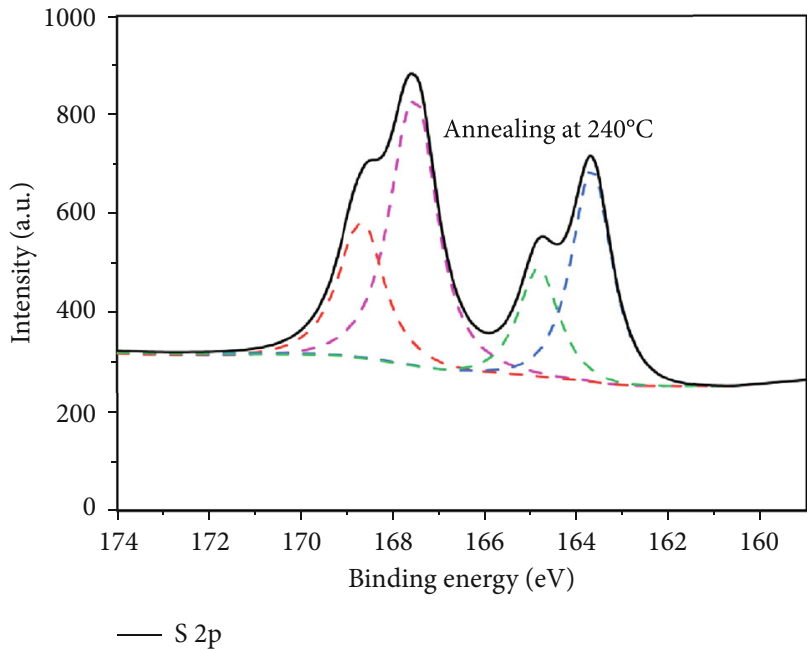

(d)

FIGURE 5: (a) XPS spectra and (b-d) its deconvolution analyses of $S$ 2p peaks for (b) the pristine PEDOT : PSS film and the film annealed at (c) 220 or (d) $240^{\circ} \mathrm{C}$.

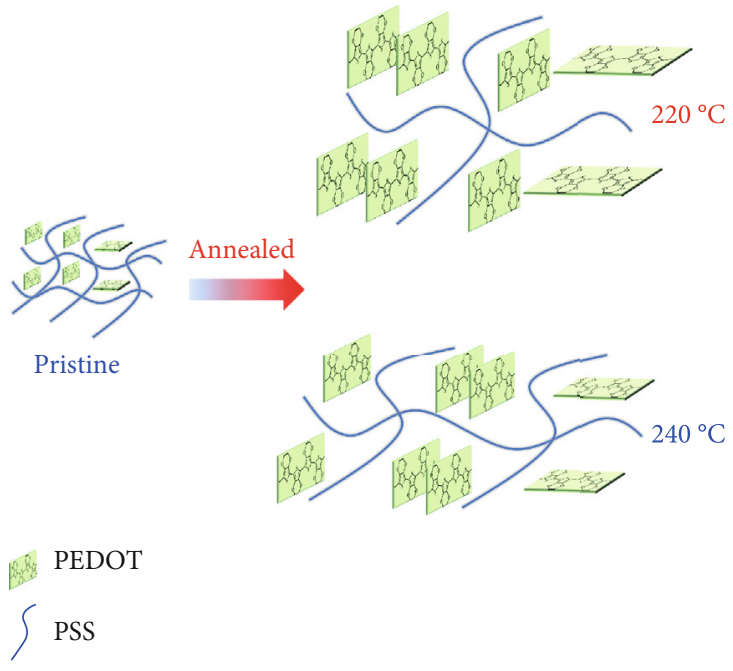

FIGURE 6: A schematic illustration showing the effect of annealing on crystalline microstructure evolution in PEDOT : PSS thick films. induced crystalline microstructure evolution on carrier transport and TE performance for PEDOT : PSS films. The annealing yields obvious changes of crystallographic and morphologic structures in the films with thickness of $\sim 10 \mu \mathrm{m}$, including improved crystallinity, enlarged crystal domain dimension, and domain morphology transformation from granule to crystalline nanofibril. In addition, $\pi-\pi$ edgeon stacking is the primary orientation, and the fraction of insulating PSS is reduced in the film skin layer. These microstructural variances lead to a mechanism transition from $3 \mathrm{D}$ to $1 \mathrm{D}$ for carrier transport, decreased activation energy, and improved mobility, hence facilitating the carrier hopping and transport. As a consequence, the TE performance enhances greatly upon annealing. Annealed at the optimized temperature of $220^{\circ} \mathrm{C}$, the film displays a significantly enhanced power factor, being 162.5 times of that of the pristine film before annealing. Based on the mechanism proposed herein, it can be envisioned that by judicious crystalline microstructure evolution, the TE performance 
for other polymers and polymer-based composites can be conveniently adjusted and remarkably enhanced. This work sheds light on the understanding of material chemistry and provides a guideline to fabricate high-performance organic and composite TE materials.

\section{Data Availability}

All data needed to evaluate the conclusions in the paper are presented in the paper and/or the Supplementary Materials. Additional data related to this paper may be requested from the authors (G.C.).

\section{Conflicts of Interest}

The authors declare no conflict of interest.

\section{Authors' Contributions}

G.C. conceived the idea and designed the experiments. X.H. conducted the experiments, TE property measurement, and characterization analysis. X.H., L.D., F.L., and Q.Z. contributed to data analysis, model interpretation, and paper drafting. X.H. and G.C. completed the writing of the manuscript. All authors discussed the results and gave comments regarding the manuscript.

\section{Acknowledgments}

G. Chen acknowledges the financial supports by the National Natural Science Foundation of China (No. 51973122). Q.Z. acknowledges financial support from AcRF Tier 1 (RG 111/17, RG 2/17, RG 114/16, and RG 8/16) and Tier 2 (MOE 2017-T2-1-021 and MOE 2018-T2-1-070), Singapore. Q.Z. also thanks the support from the State Key Laboratory of Supramolecular Structure and Materials, Jilin University (sklssm2020041).

\section{Supplementary Materials}

Materials and methods. Figure S1: preparation procedure of PEDOT : PSS films. Figure S2: the PEDOT : PSS film displays high flexible. Figure S3: TGA curve of the PEDOT : PSS film. Figure S4: temperature dependence curve of pristine PEDOT : PSS. Table S1: D-spacing, crystalline information of PEDOT : PSS annealed films (Supplementary Materials)

\section{References}

[1] L. Tzounis, M. Hegde, M. Liebscher et al., "All-aromatic SWCNT-polyetherimide nanocomposites for thermal energy harvesting applications," Composites Science and Technology, vol. 156, pp. 158-165, 2018.

[2] I. Petsagkourakis, N. Kim, K. Tybrandt, I. Zozoulenko, and X. Crispin, "Poly(3,4-ethylenedioxythiophene): chemical synthesis, transport properties, and thermoelectric devices," Advanced Electronic Materials, vol. 5, no. 11, p. 1800918, 2019.

[3] D. Qu, X. Huang, L. Li, H. Wang, and G. Chen, "NPJ Felx," Electronics, vol. 4, p. 1, 2020.
[4] G. Chen, Y. Li, M. Bick, and J. Chen, "Smart textiles for electricity generation," Chemical Reviews, vol. 120, no. 8, pp. 3668-3720, 2020.

[5] C. L. K. Cho, P. Tzeng, J.-H. Hsu, and C. Yu, “Outstanding low temperature thermoelectric power factor from completely organic thin films enabled by multidimensional conjugated nanomaterials," Advanced Energy Materials, vol. 6, no. 7, p. 1502168, 2016.

[6] L. Liang, C. Gao, G. Chen, and C.-Y. Guo, "Large-area, stretchable, super flexible and mechanically stable thermoelectric films of polymer/carbon nanotube composites," Journal of Materials Chemistry C, vol. 4, no. 3, pp. 526-532, 2016.

[7] J.-H. Bahk, H. Fang, and K. Yazawa, "Flexible thermoelectric materials and device optimization for wearable energy harvesting," Journal of Materials Chemistry C, vol. 3, no. 40, pp. 10362-10374, 2015.

[8] S. Pu, J. Fu, Y. Liao et al., "Promoting energy efficiency via a self-adaptive evaporative cooling hydrogel," Advanced Materials, vol. 32, no. 17, p. 1907307, 2020.

[9] Y. Chen, Y. Zhao, and Z. Liang, "Solution processed organic thermoelectrics: towards flexible thermoelectric modules," Energy \& Environmental Science, vol. 8, no. 2, pp. 401-422, 2015.

[10] S. Han, F. Jiao, Z. U. Khan, J. Edberg, S. Fabiano, and X. Crispin, "Thermoelectric polymer aerogels for pressuretemperature sensing applications," Advanced Functional Materials, vol. 27, no. 44, p. 1703549, 2017.

[11] G. Wu, Z.-G. Zhang, Y. Li, C. Gao, X. Wang, and G. Chen, "Exploring high-performance n-type thermoelectric composites using amino-substituted rylene dimides and carbon nanotubes," ACS Nano, vol. 11, no. 6, pp. 5746-5752, 2017.

[12] D. Qu, X. Li, H. Wang, and G. Chen, "Assembly Strategy and Performance Evaluation of Flexible Thermoelectric Devices," Advancement of Science, vol. 6, p. 1900584, 2019.

[13] Y. Xue, C. Gao, L. Liang, and X. Wang, "Nanostructure controlled construction of high-performance thermoelectric materials of polymers and their composites," Journal of Materials Chemistry A, vol. 6, no. 45, pp. 22381-22390, 2018.

[14] X. Hu, G. Chen, and X. Wang, "Tuning thermoelectric performance by nanostructure evolution of a conducting polymer," Journal of Materials Chemistry A, vol. 3, no. 42, pp. 2089620902, 2015.

[15] C. Yao, H. Zhang, and Q. Zhang, "Recent Progress in Thermoelectric Materials Based on Conjugated Polymers," Polymers, vol. 11, no. 1, p. 107, 2019.

[16] J. Wu, Y. Sun, W. Pei, L. Huang, W. Xu, and Q. Zhang, "Polypyrrole nanotube film for flexible thermoelectric application," Synthetic Metals, vol. 196, pp. 173-177, 2014.

[17] S. Zhang, Z. Fan, X. Wang, and Z. Zhang, "Enhancement of the thermoelectric properties of PEDOT:PSSviaone-step treatment with cosolvents or their solutions of organic salts," Journal of Materials Chemistry A, vol. 6, no. 16, pp. 7080-7087, 2018.

[18] S. H. Lee, H. Park, S. Kim, W. Son, I. W. Cheong, and J. H. Kim, "Transparent and flexible organic semiconductor nanofilms with enhanced thermoelectric efficiency," Journal of Materials Chemistry A, vol. 2, no. 20, pp. 7288-7294, 2014.

[19] J. Zhao and D. Tan, "A strategy to improve the thermoelectric performance of conducting polymer nanostructures," Journal of Materials Chemistry C, vol. 5, no. 1, pp. 47-53, 2017. 
[20] J. Xie, C. Zhao, Z. Lin, and P. Gu, "Nanostructured conjugated polymers for energy-related applications beyond solar cells," Chemistry, an Asian Journal, vol. 11, no. 10, pp. 1489-1511, 2016.

[21] N. Kim, S. Kee, S. H. Lee et al., "Highly Conductive PEDOT:PSS Nanofibrils Induced by Solution-Processed Crystallization," Advanced Materials, vol. 26, no. 14, pp. 2268-2272, 2014.

[22] X. Li, C. Liu, W. Zhou et al., "Roles of polyethylenimine ethoxylated in efficiently tuning the thermoelectric performance of poly(3,4-ethylenedioxythiophene)-rich nanocrystal films," ACS Applied Materials \& Interfaces, vol. 11, no. 8, pp. 8138-8147, 2019.

[23] B. Russ, A. Glaudell, J. L. Urban, M. L. Chabinyc, and R. A. Segalman, "Organic thermoelectric materials for energy harvesting and temperature control," Nature Reviews Materials, vol. 1, no. 10, p. 16050, 2016.

[24] X. Hu, G. Chen, and X. Wang, "An unusual coral-like morphology for composites of poly(3,4-ethylenedioxythiophene)/carbon nanotube and the enhanced thermoelectric performance," Composites Science and Technology, vol. 144, pp. 43-50, 2017.

[25] T. Park, C. Park, B. Kim, H. Shin, and E. Kim, "Flexible PEDOT electrodes with large thermoelectric power factors to generate electricity by the touch of fingertips," Energy \& Environmental Science, vol. 6, no. 3, pp. 788-792, 2013.

[26] L. Wang, Z. Zhang, Y. Liu et al., "Exceptional thermoelectric properties of flexible organic-inorganic hybrids with monodispersed and periodic nanophase," Nature Communications, vol. 9, no. 1, p. 3817, 2018.

[27] X. Wang, X. Zhang, L. Sun et al., "High electrical conductivity and carrier mobility in oCVD PEDOT thin films by engineered crystallization and acid treatment," Science Advances, vol. 4, no. 9, p. eaat5780, 2018.

[28] A. Ugur, F. Katmis, M. Li et al., "Low-dimensional conduction mechanisms in highly conductive and transparent conjugated polymers," Advanced Materials, vol. 27, no. 31, pp. 4604-4610, 2015.

[29] D. T. Duong, V. Ho, Z. Shang et al., "Mechanism of crystallization and implications for charge transport in poly(3-ethylhexylthiophene) thin films," Advanced Functional Materials, vol. 24, no. 28, pp. 4515-4521, 2014.

[30] L. Nian, Z. Chen, S. Herbst et al., “Aqueous solution processed photoconductive cathode interlayer for high performance polymer solar cells with thick interlayer and thick active layer," Advanced Materials, vol. 28, no. 34, pp. 7521-7526, 2016.

[31] N. Lienemann, S. Kim, I. Petsagkourakis et al., "Elastic conducting polymer composites in thermoelectric modules," Nature Communications, vol. 11, no. 1, p. 1424, 2020.

[32] Z. Li, H. Sun, C.-L. Hsiao et al., "A free-standing high-output power density thermoelectric device based on structureordered PEDOT:PSS," Advanced Electronic Materials, vol. 4, no. 2, p. 1700496, 2018.

[33] Q. Wang, C. R. Bowen, R. Lewis et al., "Hexagonal boron nitride nanosheets doped pyroelectric ceramic composite for high-performance thermal energy harvesting," Nano Energy, vol. 60, pp. 144-152, 2019.

[34] S. Pu, Y. Liao, K. Chen et al., "Thermogalvanic hydrogel for synchronous evaporative cooling and low-grade heat energy harvesting," Nano Letters, vol. 20, no. 5, pp. 3791-3797, 2020.
[35] B. Friedel, P. E. Keivanidis, T. J. K. Brenner et al., "Effects of layer thickness and annealing of PEDOT:PSS layers in organic photodetectors," Macromolecules, vol. 42, no. 17, pp. 67416747, 2009.

[36] G. Greczynski, T. Kugler, and W. R. Salaneck, "Characterization of the PEDOT-PSS system by means of X-ray and ultraviolet photoelectron spectroscopy," Thin Solid Films, vol. 354, no. 1-2, pp. 129-135, 1999.

[37] K. Wilson, A. F. Lee, and D. J. Macquarrie, "Structure and reactivity of sol-gel sulphonic acid silicas," Applied Catalysis A: General, vol. 228, no. 1-2, pp. 127-133, 2002.

[38] R. Kiebooms, A. Aleshin, K. Hutchison, F. Wudl, and A. Heeger, "Doped poly(3,4-ethylenedioxythiophene) films: thermal, electromagnetical and morphological analysis," Synthetic Metals, vol. 101, no. 1-3, pp. 436-437, 1999.

[39] J. Huang, P. F. de Miller, J. C. de Mello, A. J. Mello, and D. D. C. Bradley, "Influence of thermal treatment on the conductivity and morphology of PEDOT/PSS films," Synthetic Metals, vol. 139, no. 3, pp. 569-572, 2003.

[40] W. Ma, C. Yang, X. Gong, K. Lee, and A. J. Heeger, "Thermally stable, efficient polymer solar cells with nanoscale control of the interpenetrating network morphology," Advanced Functional Materials, vol. 15, no. 10, pp. 1617-1622, 2005.

[41] J. Luo, D. Billep, T. Waechtler et al., "Enhancement of the thermoelectric properties of PEDOT:PSS thin films by post-treatment," Journal of Materials Chemistry A, vol. 1, no. 26, pp. 7576-7583, 2013.

[42] S. Ashizawa, R. Horikawa, and H. Okuzaki, "Effects of solvent on carrier transport in poly(3,4-ethylenedioxythiophene)/poly(4-styrenesulfonate)," Synthetic Metals, vol. 153, no. 1-3, pp. 5-8, 2005.

[43] A. M. Nardes, R. A. J. Janssen, and M. Kemerink, "A morphological model for the solvent-enhanced conductivity of PEDOT:PSS thin films," Advanced Functional Materials, vol. 18, no. 6, pp. 865-871, 2008.

[44] V. K. Shante and C. M. Varma, "Hopping conductivity in "one-dimensional" disordered compounds," Physical Review B, vol. 8, no. 10, pp. 4885-4889, 1973.

[45] C. M. Palumbiny, F. Liu, T. P. Russell, A. Hexemer, C. Wang, and P. Muller-Buschbaum, "The crystallization of PEDOT:PSS polymeric electrodes probed in situ during printing," Advanced Materials, vol. 27, no. 22, pp. 3391-3397, 2015.

[46] J. Rivnay, S. Inal, B. A. Collins et al., "Structural control of mixed ionic and electronic transport in conducting polymers," Nature Communications, vol. 7, no. 1, p. 11287, 2016.

[47] Y. Xia, K. Sun, and J. Ouyang, "Solution-processed metallic conducting polymer films as transparent electrode of optoelectronic devices," Advanced Materials, vol. 24, no. 18, pp. 24362440, 2012.

[48] G. H. Kim, L. Shao, K. Zhang, and K. P. Pipe, "Engineered doping of organic semiconductors for enhanced thermoelectric efficiency," Nature Materials, vol. 12, no. 8, pp. 719-723, 2013.

[49] K. Kang, S. Watanabe, K. Broch et al., "2D coherent charge transport in highly ordered conducting polymers doped by solid state diffusion," Nature Materials, vol. 15, no. 8, pp. 896-902, 2016.

[50] T. Takano, H. Masunaga, A. Fujiwara, H. Okuzaki, and T. Sasaki, "PEDOT nanocrystal in highly conductive PEDOT:PSS polymer films," Macromolecules, vol. 45, no. 9, pp. 3859-3865, 2012. 